\title{
Triples of Orthogonal Latin and Youden Rectangles For Small Orders
}

\author{
Gerold Jäger \\ gerold.jager@umu.se \\ Denys Shcherbak \\ denys.shcherbak@umu.se
}

\author{
Klas Markström \\ klas.markstrom@umu.se \\ Lars-Daniel Öhman \\ lars-daniel.ohman@umu.se
}

October 31, 2018

\begin{abstract}
We have performed a complete enumeration of non-isotopic triples of mutually orthogonal $k \times n$ Latin rectangles for $k \leq n \leq 7$. Here we will present a census of such triples, classified by various properties, including the order of the autotopism group of the triple. As part of this we have also achieved the first enumeration of pairwise orthogonal triples of Youden rectangles. We have also studied orthogonal triples of $k \times 8$ rectangles which are formed by extending mutually orthogonal triples with non-trivial autotopisms one row at a time, and requiring that the autotopism group is non-trivial in each step. This class includes a triple coming from the projective plane of order 8 . Here we find a remarkably symmetrical pair of triples of $4 \times 8$ rectangles, formed by juxtaposing two selected copies of complete sets of MOLS of order 4 .
\end{abstract}

\section{Introduction}

Sets of mutually orthogonal Latin squares (MOLS) are well studied objects in both pure combinatorics and statistical design theory, see e.g. Chapter 10 of HK08, with a history going at least all the way back to Euler's 36 officers problem and the conjectures he made based on his studies of that problem. In the present paper we will study a variation on this topic, namely sets of mutually orthogonal Latin rectangles. For $k \leq n$, a Latin rectangle is a $k \times n$ array using $n$ different symbols, such that each row is a permutation of the symbols, and all symbols in a column are distinct. In particular, an $n \times n$ Latin rectangle is a Latin square. The concept of orthogonality in Latin squares can be naturally extended to rectangles by saying that the rectangles $R$ and $L$ are orthogonal if all the ordered pairs of symbols $\left(R_{i j}, L_{i j}\right)$ are distinct. More specifically, the aim has been to classify all non-isotopic triples of MOLS and mutually orthogonal Latin rectangles (MOLR) for as large $n$ as possible, which turns out to be $n=7$. 
One reason for choosing to work with triples is a fundamental question in the theory of mutually orthogonal Latin squares: How large can a set of MOLS be for a given $n$ ? The maximum size of such a set is here denoted by $N(n)$. Thanks to the now classical results by Bose, Tarry and others Bos38, BSP60, Tar00 we know that for $n \neq 2,6$ there exist MOLS, i.e. $N(n) \geq 2$ for $n \neq 2,6$. It was also proven by Chowla, Erdős and Straus [CES60 that there exist constants $C, \alpha>0$ such that $N(n) \geq C n^{\alpha}$ for large enough $n$. This result has been improved in various ways over the years and recently Barber, Kühn, Lo, Osthus and Taylor $\mathrm{BKL}^{+} 17$ proved that one can prescribe a substantial number of entries in $r$ partial Latin squares and still be able to extend these partial Latin squares to a set of $r$ MOLS, where $r$ grows with $n$.

For small $n$, a sequence of results, culminating with Todorov Tod85, lead to the result that $N(n) \geq 3$ unless $n=2,3,6$ and possibly $n=10$. The open case $n=10$ is of special interest due to its connection to the existence of finite projective planes; a finite projective plane of order $n$ is equivalent to a set of $n-1$ MOLS. It is believed that a projective plane only exists for orders which are prime powers and the first open case for the conjecture was for a long time $n=10$. This case was settled in 1989 [LTS89 by a combination of methods from coding theory and an extensive computer search, an effort well surveyed in Lam91. That result in fact shows that $N(10) \leq 6$, but the result would of course itself follow if in fact $N(10)=2$. A number of authors have attempted to construct triples of MOLS for $n=10$ and the latest large scale such effort MMM07 proved that if such a triple exists, then each of the three Latin squares involved must have a trivial symmetry group. For Latin rectangles it is however trivial that triples of orthogonal $1 \times n$ rectangles exist, and it is not hard to show that triples of mutually orthogonal $2 \times n$ rectangles exist if $n \geq 3$, as noted in Agg75. One reason for studying triples of MOLR is therefore that if there is no triple of MOLS of order 10, there must be a largest $k<10$ such that a triple of mutually orthogonal $k \times 10$ rectangles does exist.

In a more general perspective, with the double aim of finding rectangular orthogonal arrays for design purposes and getting a better understanding of the existence of MOLS we have generated and classified all orthogonal triples of MOLR up to $n=7$. For each fixed $n$, the number of non-isotopic $k \times n$ triples turns out to be a unimodal function of $k$, and even for $n=6$, where no pair of orthogonal Latin squares exists, there are several examples of triples of MOLR with $k=5$. For each value of $n$ we have also counted the number of non-isotopic maximal triples, i.e., triples which cannot be extended by one more row. These results are described in Subsection 4.1. Further, as described in Subsection 4.3 we have computed the autotopism groups of the orthogonal triples. Here we see a clear change in behaviour when $n$ increases. For $n \leq 5$ the autotopism groups are always non-trivial, but at $n=6$ we see the first examples with no autotopisms, and for $n=7$ this is the dominant case.

The paper is structured as follows. In Section 2 we give notation and formal definitions. In Section 3 we state the questions guiding our investigation, describe briefly the algorithm used to find all triples of MOLR and give some practical information regarding the computer calculations. In Subsection 4.1 
we present the data our computer search resulted in, in particular the number of non-isotopic triples of MOLR for each order. By means of analysis of the data produced, we discuss the extendability of the triples. In Subsection 4.2 we discuss which of the triples found satisfy the stronger condition of being Youden rectangles and in Subsection 4.3 we discuss the autotopism groups of the triples. Up to order $n=7$ our enumeration is complete, and in Section 5 we discuss our enumeration of triples of order $n=8$ with the added condition that they sequentially for each $k$ have non-trivial autotopism groups.

\section{Notation and Definitions}

A Latin square is an $n \times n$ matrix with cells filled by $n$ symbols such that each row and each column contains a specific symbol exactly once. For $k \leq n$ a matrix with $k$ rows and $n$ columns whose cells are filled by $n$ symbols such that each row contains each symbol exactly once and each column contains each symbol at most once is called a $k \times n$ Latin rectangle. In the following we use as symbol set $\{0,1, \ldots, n-1\}$. We denote the $t$-th row of a $k \times n$ rectangle $A$ by $A_{t}$ for $t \in\{1,2, \ldots, k\}$.

Let $A=\left(\alpha_{i, j}\right)_{1 \leq i, j, \leq n}$ and $B=\left(\beta_{i, j}\right)_{1 \leq i, j, \leq n}$ be Latin squares. We say that $A$ and $B$ are mutually orthogonal Latin squares if the set of ordered pairs $\left\{\left(\alpha_{i, j}, \beta_{i, j}\right) \mid i, j \in\{1,2 \ldots n\}\right\}$ contains all possible ordered pairs, or, in other words, if each ordered pair $\left(\alpha_{i, j}, \beta_{i, j}\right)$ appears exactly once. A set of Latin squares of order $n$ is called a set of Mutually Orthogonal Latin Squares (MOLS) if each square is orthogonal to every other square in the set. Similarly, we can extend the orthogonality condition to Latin rectangles. We say that Latin rectangles of size $k \times n A=\left(\alpha_{i, j}\right)_{1 \leq i \leq k, 1 \leq j \leq n}$ and $B=\left(\beta_{i, j}\right)_{1 \leq i \leq k, 1 \leq j \leq n}$ are orthogonal if each ordered pair $\left(\alpha_{i, j}, \beta_{i, j}\right)$ appears at most once. Also, a set of pairwise orthogonal Latin rectangles is called a set of Mutually Orthogonal Latin Rectangles (MOLR).

There are many different notions of 'equivalence' of Latin squares, Latin rectangles, mutually orthogonal Latin squares, and mutually orthogonal Latin rectangles (see, for example, [LM98 and [EW16]). In the present paper, we use the equivalence notion isotopism: Two triples $T_{1}$ and $T_{2}$ of MOLR are said to be isotopic if $T_{2}$ can be gotten from $T_{1}$ by permuting the order of the three rectangles, by permuting rows (jointly in all three rectangles in $T_{1}$ ), permuting columns (jointly in all three rectangles in $T_{1}$ ), and by permuting symbols (separately in each of the three rectangles in $T_{1}$ ). It should be noted that all these permutations will preserve the orthogonality condition, and that there are no further obvious transformations that will.

Considering that our main focus in the present paper is Latin rectangles, we have chosen not to use a stronger concept of 'equivalence' sometimes used for Latin squares, where interchanges of the roles of rows, columns, and symbols are also allowed. In a Latin rectangle, rows, columns and symbols do not play the same role, so such interchanges would not result in Latin rectangles. In the special case of $k=n$, which we also study, applying this stronger notion 
of 'equivalence' is meaningful, and would result in so-called main classes, or species, of MOLS. This enumeration, however, has already been done in EW16] for all the $n$ included in our study, and so we do not repeat it here.

We will also be interested in how many triples of MOLR are isotopic, which motivates the following slightly different presentation and additional terminology. Let $(A, B, C)$ be a triple of MOLR of size $k \times n$. The following group of isotopisms acts on the set of triples of MOLR: $G_{n, k}=S_{3} \times S_{k} \times S_{n} \times\left[S_{n} \times S_{n} \times S_{n}\right]$, where $S_{3}$ corresponds to a permutation of the rectangles, $S_{k}$ corresponds to a permutation of the rows, $S_{n}$ corresponds to a permutation of the columns, and each of the last three $S_{n}$ correspond to a permutation of the symbols in a single rectangle. Two triples $(A, B, C)$ and $\left(A^{\prime}, B^{\prime}, C^{\prime}\right)$ of MOLR of size $k \times n$ are isotopic if there exists a $g \in G_{n, k}$ such that $g(A, B, C)=\left(A^{\prime}, B^{\prime}, C^{\prime}\right)$. The autotopism group of a triple $(A, B, C)$ is defined as $\operatorname{Aut}(A, B, C):=\{g \in$ $\left.G_{n, k} \mid g(A, B, C)=(A, B, C)\right\}$.

Since each row of a Latin square or a Latin rectangle can be seen as a permutation, sometimes we refer to rows as permutations. We say that a permutation $\sigma \in S_{n}$, where $S_{n}$ denotes the symmetric group on $n$ elements, is lexicographically smaller than a permutation $\pi \in S_{n}$ and write $\sigma<\pi$, if $\sigma(i)<\pi(i)$, where $i$ is the first position with $\sigma(i) \neq \pi(i)$. The lexicographical comparison can be extended to triples of MOLR as follows. Let $(A, B, C)$ and $\left(A^{\prime}, B^{\prime}, C^{\prime}\right)$ be triples of $k \times n$ MOLR. Furthermore, let $A, B$ and $C$ consist of permutations $\sigma_{i, s}$, and $A^{\prime}, B^{\prime}, C^{\prime}$ consist of permutations $\pi_{i, s}$, respectively, where $\sigma_{i, s}, \pi_{i, s} \in S_{n}$ for $i \in\{1,2 \ldots k\}$ and $s \in\{1,2,3\}$. The sequence of triples of permutations $\left\{\left(\sigma_{1,1}, \sigma_{1,2}, \sigma_{1,3}\right),\left(\sigma_{2,1}, \sigma_{2,2}, \sigma_{2,3}\right), \ldots,\left(\sigma_{k, 1}, \sigma_{k, 2}, \sigma_{k, 3}\right)\right\}$ describes $(A, B, C)$ and $\left\{\left(\pi_{1,1}, \pi_{1,2}, \pi_{1,3}\right),\left(\pi_{2,1}, \pi_{2,2}, \pi_{2,3}\right), \ldots,\left(\pi_{k, 1}, \pi_{k, 2}, \pi_{k, 3}\right)\right\}$ describes $\left(A^{\prime}, B^{\prime}, C^{\prime}\right)$. We say that a triple of rectangles $(A, B, C)$ is lexicographically smaller than $\left(A^{\prime}, B^{\prime}, C^{\prime}\right)$ if $\sigma_{i, s}<\pi_{i, s}$, where $i, s$ are the first indices in the sequences with $\sigma_{i, s} \neq \pi_{i, s}$. In other words, we compare triples of MOLR by rows.

We call a triple of MOLR normalized if it satisfies the following conditions:

(S1) (Ordering among columns) The first row of each rectangle is the identity permutation.

(S2) (Ordering among rectangles) The second row of the first rectangle is lexicographically larger than the second row of the second rectangle, and the second row of the second rectangle is larger than the second row of the third rectangle. In other words, if $a_{1}, a_{2}, a_{3}$ are symbols on the positions $(2,1)$ in the ordered triple, then it holds that $a_{1}>a_{2}>a_{3}$. Note that $a_{1}, a_{2}, a_{3}$ are pairwise distinct, since all the ordered pairs with the same symbol in each position occur in the first row.

(S3) (Ordering among rows) The second row in the first rectangle is lexicographically larger than the third one, the third one is larger than the fourth one, and so on.

In Figure 1 we give an example of a normalized triple of MOLS. 


\begin{tabular}{|l|l|l|l|}
\hline 0 & 1 & 2 & 3 \\
\hline 3 & 2 & 1 & 0 \\
\hline 2 & 3 & 0 & 1 \\
\hline 1 & 0 & 3 & 2 \\
\hline
\end{tabular}

\begin{tabular}{|l|l|l|l|}
\hline 0 & 1 & 2 & 3 \\
\hline 2 & 3 & 0 & 1 \\
\hline 1 & 0 & 3 & 2 \\
\hline 3 & 2 & 1 & 0 \\
\hline
\end{tabular}

\begin{tabular}{|l|l|l|l|}
\hline 0 & 1 & 2 & 3 \\
\hline 1 & 0 & 3 & 2 \\
\hline 3 & 2 & 1 & 0 \\
\hline 2 & 3 & 0 & 1 \\
\hline
\end{tabular}

Figure 1: A normalized triple of MOLS of order 4.

Finally, as our computations proceed by adding consecutive rows to triples of Latin rectangles, we will have use for the following term: An extension of size $k \times n$ is a triple of MOLR which results from a triple of MOLR of size $(k-1) \times n$ by adding one more row.

\section{Generating data}

\subsection{Guiding questions}

Our approach is complete enumeration by computer for as large parameters as possible, and unless otherwise stated, we save all generated data. In particular, we do not only record the number of triples of MOLR found, but with some exceptions noted below, we save the triples of MOLR themselves.

With some size exceptions due to size restrictions, the data generated is available for download at Web]. Further details are given there.

The following questions serve as guides for which data to generate.

(Q1) How many normalized $k \times n$ triples of MOLR are there?

(Q2) How many isotopism classes of $k \times n$ triples of MOLR are there?

(Q3) How many non-isotopic $k \times n$ triples of MOLR are maximal, i.e. cannot be extended by one more row?

(Q4) Are there sets of triples of MOLR that satisfy some stronger regularity conditions? In particular, are any of the triples of MOLR in fact triples of Youden rectangles?

(Q5) Which order does the autotopism group of each $k \times n$ triple of MOLR have?

We note that it is clear from the definitions that the number of non-isotopic triples of MOLR will be less than the number of normalized ones.

\subsection{Algorithms}

We employ two main algorithms.

1. Extension Finding: This first algorithm finds $k \times n$ triples of MOLR as extensions of all non-isotopic $(k-1) \times n$ triples of MOLR and counts the number of instances which are maximal. 
2. Isotopism Rejection: This algorithm applies an isotopism rejection for $k \times n$ triples of MOLR, keeping one representative of each isotopism class. This algorithm also keeps track of the order of the autotopism groups.

Pseudocode and more detailed explanations of the algorithms are given in Appendix F.1 and Appendix F.2, respectively.

\subsection{Implementation and Execution}

We use Algorithms 1 and 2 in the following two computations. First, we find all normalized $k \times n$ triples of MOLR. More precisely, we count the number of normalized $k \times n$ triples of MOLR, the number of isotopism classes and the number of $k \times n$ triples of MOLR which are maximal (thus addressing questions (Q1), (Q2) and (Q3)). Second, we classify the triples of MOLR according to the order of their autotopism group (thus addressing question (Q5)). Checking the Youden property (question (Q4)) was done by a separate, simple program.

We have implemented the algorithms in $\mathrm{C}++$. Each classification was done for $n=4,5,6,7$ and $k \in\{2,3, \ldots, n\}$. The computation for $n=4,5,6$ was easily done on a standard desktop. For $n \geq 7$ both the computational effort and the disc requirements were significantly larger. We therefore parallelized Algorithms 1 and 2 and ran the experiments on the parallel machine Kebnekaise from the High Performance Computing Center North (HPC2N).

The running time of the first computation on a standard desktop is less than one minute for all $n=4,5,6$. For $n=7$ the number of triples of MOLR is greater than 1.4 billion, with more than 400 million isotopism classes, and the running time of both the programs (generation and isotopism reduction) is almost 200 core hours. The running time for the computation of the autotopism groups is similar. It can be done in one minute for all sizes except $3 \times 7$ and $4 \times 7$, where the program requires about 80 core hours.

As will be described in Section 5 , the parallelized version of the algorithms were also used to study the number of $3 \times 8$ triples of MOLR.

\section{Results and Analysis}

We now turn to the results and analysis of our computational work.

\subsection{The Number of Triples of MOLR}

Our first result is an enumeration of triples of MOLR. Table 1 lists the number of normalized $k \times n$ triples, the number of isotopism classes and how many of the non-isotopic cases are maximal, i.e., cannot be extended by one more row.

In appendix $\mathrm{A}$ the unique (up to isotopism) $4 \times 4$ triple is given, in appendix $B$ the unique (up to isotopism) $5 \times 5$ triple is given, in appendix $\mathrm{C}$, the 7 non-isotopic $5 \times 6$ triples are given, and in appendix D, the 4 non-isotopic $7 \times 7$ triples are given. 


\begin{tabular}{|r|r|r|r|}
\hline \multirow{2}{*}{ Size } & \multirow{2}{*}{ \#Normalized } & \multicolumn{2}{|c|}{ \#Non-isotopic } \\
\cline { 3 - 4 } & & \multicolumn{1}{|c|}{ \#Total } & \#Maximal \\
\hline $2 \times 4$ & 4 & 2 & 1 \\
\hline $3 \times 4$ & 2 & 1 & 0 \\
\hline $4 \times 4$ & 1 & 1 & - \\
\hline \hline $2 \times 5$ & 224 & 4 & 3 \\
\hline $3 \times 5$ & 3 & 1 & 0 \\
\hline $4 \times 5$ & 2 & 1 & 0 \\
\hline $5 \times 5$ & 1 & 1 & - \\
\hline \hline $2 \times 6$ & 65520 & 103 & 0 \\
\hline $3 \times 6$ & 16767 & 2572 & 1800 \\
\hline $4 \times 6$ & 2005 & 513 & 493 \\
\hline $5 \times 6$ & 31 & 7 & 7 \\
\hline $6 \times 6$ & 0 & 0 & - \\
\hline \hline $2 \times 7$ & 25864320 & 2858 & 0 \\
\hline $3 \times 7$ & 200127181 & 65883453 & 30025 \\
\hline $4 \times 7$ & 1292959311 & 323112477 & 322850101 \\
\hline $5 \times 7$ & 273190 & 55545 & 55508 \\
\hline $6 \times 7$ & 42 & 16 & 12 \\
\hline $7 \times 7$ & 4 & 4 & - \\
\hline
\end{tabular}

Table 1: The number of orthogonal $k \times n$ triples.

From Table 1 we observe that the behavior of the number of non-isotopic triples and the total number of triples is similar. The number of non-isotopic triples increases with $k$ up to around $k \approx \frac{n}{2}$ and the increase is very fast, but in the steps after the peak the numbers rapidly decrease. There are at least two reasons for this decrease of the number of triples of MOLR. First, the orthogonality condition conflicts with the Latin rectangle condition. As a result orthogonality fails on some positions, and this becomes more frequent when $k$ increases. Second, the number of non-isotopic triples decreases because of isotopic extensions, i.e., non-isotopic triples sometimes produce isotopic extensions.

In Example 1we show a maximal triple of MOLR. This example is interesting since $n=5$ is the largest order where we find maximal triples with just two rows.

Example 1. A maximal $2 \times 5$ triple of MOLR:

\begin{tabular}{|l|l|l|l|l|}
\multicolumn{5}{c}{$A$} \\
\hline 0 & 1 & 2 & 3 & 4 \\
\hline 4 & 3 & 1 & 2 & 0 \\
\hline
\end{tabular}

\begin{tabular}{|l|l|l|l|l|}
\multicolumn{7}{|c|}{$B$} \\
\hline 0 & 1 & 2 & 3 & 4 \\
\hline 3 & 4 & 0 & 1 & 2 \\
\hline
\end{tabular}

\begin{tabular}{|l|l|l|l|l|}
\multicolumn{5}{c}{$C$} \\
\hline 0 & 1 & 2 & 3 & 4 \\
\hline 2 & 0 & 3 & 4 & 1 \\
\hline
\end{tabular}

Each of these rectangles can be extended to Latin rectangles with 3 rows by 12, 13, 13 permutations, respectively, but the orthogonality condition cannot be satisfied. 
In general, the number of rows in a maximal triple must grow with $n$, as the following proposition shows. In particular, it follows from this result that there are no maximal triples of MOLR of order $2 \times 6$ or $2 \times 7$.

Proposition 4.1. A triple of $k \times n M O L R$ is not maximal if $k \leq \frac{n-1}{3}$.

Proof. Assume that the triple consists of the three rectangles $R_{1}, R_{2}, R_{3}$. We want to show that under the condition on $k$ we can extend each rectangle by one row, while preserving orthogonality. We define bipartite graphs $G_{i}$, one for each rectangle, which has $n$ vertices which correspond to the columns of $R_{i}$ and $n$ vertices which correspond to the symbols of $R_{i}$. The edges of the graph are given as follows: We start out with all edges with one endpoint in each vertex class and then delete every edge $(c, s)$ where the column $c$ contains the symbol $s$. Here each row of $R_{i}$ leads to the deletion of a perfect matching, so $R_{i}$ is a regular graph with vertex degree $n-k$.

Now, since $G_{1}$ is regular and bipartite, it has a perfect matching $M_{1}$, and we will use the corresponding assignment of symbols as the new row $r_{1}$ in $R_{1}$. The new row assigns symbols to each position, and for each symbol there are now $k$ symbols which are forbidden in that position in the other two rectangles because of the orthogonality condition. These are the symbols which appear in symbol pairs in earlier rows.

We delete those edges from $G_{2}$ and $G_{3}$ to get the graphs $G_{2}^{\prime}$ and $G_{3}^{\prime}$. Each symbol appears in the same number of pairs so the edges deleted due to orthogonality induce a $k$-regular graph, this means that $G_{2}^{\prime}$ and $G_{3}^{\prime}$ have degree at least $n-2 k$ and still satisfy Hall's condition if this is at least 1 , and so we can find a perfect matching in $G_{2}^{\prime}$ which gives us a valid new row $r_{2}$ for $R_{2}$.

We can now repeat this for $G_{3}$ as long as $n-3 k \geq 1$, which is equivalent to our assumption $k \leq \frac{n-1}{3}$.

It is clear that the proof idea in this proposition can be extended to give a similar result for extendability of $m$-tuples of MOLR, but that is not of interest in the present paper.

Despite the fact that some triples of rectangles cannot be extended by one extra row because of orthogonality issues, sometimes it is possible to partially extend them, in other words, to simultaneously fill some position in the next row of each rectangle while satisfying the Latin rectangle condition and the orthogonality condition. However, there are examples where we cannot simultaneously fill any position in the next row, as demonstrated in Example 2.

Example 2. Consider the following $5 \times 6$ triple of MOLR and the unique extensions of each rectangle to a Latin square: 


\begin{tabular}{|l|l|l|l|l|l|}
\multicolumn{7}{|c}{$A$} \\
\hline 0 & 1 & 2 & 3 & 4 & 5 \\
\hline 5 & 4 & 3 & 2 & 1 & 0 \\
\hline 4 & 5 & 1 & 0 & 3 & 2 \\
\hline 3 & 2 & 5 & 4 & 0 & 1 \\
\hline 2 & 0 & 4 & 1 & 5 & 3 \\
\hline \hline $\mathbf{1}$ & $\mathbf{3}$ & $\mathbf{0}$ & $\mathbf{5}$ & $\mathbf{2}$ & $\mathbf{4}$ \\
\hline
\end{tabular}

\begin{tabular}{|l|l|l|l|l|l|}
\multicolumn{7}{c}{$B$} \\
\hline 0 & 1 & 2 & 3 & 4 & 5 \\
\hline 4 & 5 & 1 & 0 & 3 & 2 \\
\hline 3 & 2 & 5 & 4 & 0 & 1 \\
\hline 2 & 4 & 3 & 1 & 5 & 0 \\
\hline 5 & 3 & 0 & 2 & 1 & 4 \\
\hline \hline $\mathbf{1}$ & $\mathbf{0}$ & $\mathbf{4}$ & $\mathbf{5}$ & $\mathbf{2}$ & $\mathbf{3}$ \\
\hline
\end{tabular}

\begin{tabular}{|l|l|l|l|l|l|}
\multicolumn{8}{|c}{$C$} \\
\hline 0 & 1 & 2 & 3 & 4 & 5 \\
\hline 3 & 2 & 5 & 4 & 0 & 1 \\
\hline 5 & 0 & 4 & 2 & 1 & 3 \\
\hline 4 & 5 & 1 & 0 & 3 & 2 \\
\hline 1 & 4 & 3 & 5 & 2 & 0 \\
\hline \hline $\mathbf{2}$ & $\mathbf{3}$ & $\mathbf{0}$ & $\mathbf{1}$ & $\mathbf{5}$ & $\mathbf{4}$ \\
\hline
\end{tabular}

We can now check the orthogonality condition, by checking the orthogonality of each pair of squares. For example, the symbol pair $(1,1)$ in the first position of the last row of the pair $(A, B)$ already appears in the first row, the symbol pair $(1,2)$ in the first position of the last row of the pair $(A, C)$ already appears in the fourth row and the symbol pair $(1,2)$ in the first position of the last row of the pair $(B, C)$ already appears in the fifth row.

We conclude that the orthogonality fails on the first position in each pair of squares. Similarly, it can be checked that orthogonality fails in each of the positions in the last row.

In fact this particularly strong form of maximality occurs in all seven instances of triples of MOLR of size $5 \times 6$ (see Appendix $\mathrm{C}$ for these seven instances). The number of $5 \times 6$ non-isotopic triples is noteworthy, since it is known that there is no pair of orthogonal Latin squares of order 6 . However, as we have seen, we can find three squares such that the failure of orthogonality can be confined to the last row of all squares.

We also note that the $7 \times 7$ orthogonal triples are interesting, as this is the first case where there is more than one non-isotopic triple of MOLS. The number of non-isotopic $7 \times 7$ orthogonal triples found coincides with the corresponding result by Egan and Wanless [EW16], where a complete enumeration of $t$-tuples of MOLS up to order 9 is given. This can be taken as an independent indication that our code is correct.

\subsection{Orthogonal Youden Rectangles}

Youden You37 introduced a class of particularly well balanced Latin rectangles, now known as either Youden rectangles or Youden "squares". A Latin rectangle is a Youden rectangle with parameter $\lambda_{c c}$ if every pair of columns has a constant number $\lambda_{c c}$ of symbols in common. As is well known, divisibility considerations immediately imply that for a $k \times n$ Youden rectangle, the parameter $\lambda_{c c}$ must satisfy $\lambda_{c c}=\frac{k(k-1)}{n-1}$. For fixed $k$ and $n$, there is therefore only one possible value of $\lambda_{c c}$, which has to be an integer.

Here we will also consider a relaxed version of this concept: a Latin rectangle is a partially balanced Youden rectangle with parameter $\lambda_{c c}^{p}>0$ if $\lambda_{c c}^{p}$ is the maximum integer such that every pair of columns intersect in at least $\lambda_{c c}^{p}$ symbols. Unlike for Youden rectangles, for partially balanced Youden rectangles the parameter $\lambda_{c c}^{p}$ is not determined uniquely by $k$ and $n$. The pigeonhole principle gives a lower bound of $2 k-n$ for $\lambda_{c c}^{p}$ so that, for example, in a $3 \times 4$ array 
columns intersect in at least 2 symbols, and in a $5 \times 7$ array columns intersect in at least 3 symbols. When the parameter $\lambda_{c c}$ is defined, that is, when $\frac{k(k-1)}{n-1}$ is an integer, it gives an upper bound for $\lambda_{c c}^{p}$, and in general, $\lambda_{c c}^{p} \leq \frac{k(k-1)}{n-1}$. When, for some order, this upper and lower bound only leave one possible value for $\lambda_{c c}^{p}$, all Latin rectangles of this order will be partially balanced with this parameter.

Sets of mutually orthogonal Youden rectangles should not be confused with multi-layered Youden rectangles, special cases of which are known as double Youden rectangles and triple Youden rectangles, studied in Cla63, Pre66, Cla67, PVP99, and PM17, but seem to be of independent interest as designs.

As far as we know this is the first complete enumeration of mutually orthogonal Youden rectangles. In Table 2 we display the number of partially balanced Youden rectangles and Youden rectangles for $n$ up to 7 . We note that removing a row from an $n \times n$ Latin square always produces an $(n-1) \times n$ Youden rectangle, so the entries in those positions in the table should come as no surprise, given the corresponding values in Table 1 We have included them in this table for completeness, and they also give an indication of the correctness of our check of the Youden property, since we actually ran the check even for these orders.

For most $k, n$ only one parameter value is possible for partially balanced Youden rectangles, and thus all Latin rectangles of these orders will be partially balanced Youden rectangles, which gives independent corroboration of the correctness of the check of the partially balanced Youden property. For the partially balanced $4 \times 7$ rectangles the three rectangles can have different parameter values and we display counts for the different combinations.

\begin{tabular}{|r|r|r|r|r|}
\hline \multirow{2}{*}{ Size } & \multicolumn{2}{|c|}{ Partially balanced } & \multicolumn{2}{|c|}{ Youden rectangles } \\
\cline { 2 - 5 } & $\#$ & $\lambda_{c c}^{p}$ & $\#$ & $\lambda_{c c}$ \\
\hline $3 \times 4$ & 1 & 2 & 1 & 2 \\
\hline \hline $3 \times 5$ & 1 & 1 & 0 & - \\
\hline $4 \times 5$ & 1 & 3 & 1 & 3 \\
\hline \hline $3 \times 6$ & 34 & 1 & 0 & - \\
\hline $4 \times 6$ & 513 & 2 & 0 & - \\
\hline $5 \times 6$ & 7 & 4 & 7 & 4 \\
\hline \hline $3 \times 7$ & 8 & 1 & 8 & 1 \\
\hline \multirow{4}{*}{$4 \times 7$} & 321312841 & $(1,1,1)$ & 0 & - \\
\cline { 2 - 5 } & 1795612 & $(1,1,2)$ & 0 & - \\
\cline { 2 - 5 } & 3993 & $(1,2,2)$ & 0 & - \\
\hline $5 \times 7$ & 31 & $(2,2,2)$ & 31 & 2 \\
\hline $6 \times 7$ & 55545 & 3 & 0 & - \\
\hline
\end{tabular}

Table 2: The number of partially balanced Youden rectangles and Youden rectangles for small $n$.

In Figure 2 we display one of the mutually orthogonal triples of $4 \times 7$ Youden 
rectangles.

\begin{tabular}{|l|l|l|l|l|l|l|}
\hline 0 & 1 & 2 & 3 & 4 & 5 & 6 \\
\hline 6 & 5 & 4 & 2 & 3 & 1 & 0 \\
\hline 3 & 4 & 1 & 6 & 0 & 2 & 5 \\
\hline 1 & 3 & 6 & 5 & 2 & 0 & 4 \\
\hline
\end{tabular}

\begin{tabular}{|l|l|l|l|l|l|l|}
\hline 0 & 1 & 2 & 3 & 4 & 5 & 6 \\
\hline 5 & 6 & 3 & 4 & 1 & 0 & 2 \\
\hline 6 & 2 & 5 & 0 & 3 & 1 & 4 \\
\hline 3 & 0 & 1 & 2 & 6 & 4 & 5 \\
\hline
\end{tabular}

\begin{tabular}{|l|l|l|l|l|l|l|}
\hline 0 & 1 & 2 & 3 & 4 & 5 & 6 \\
\hline 4 & 2 & 1 & 6 & 0 & 3 & 5 \\
\hline 5 & 6 & 0 & 1 & 2 & 4 & 3 \\
\hline 6 & 4 & 5 & 0 & 3 & 1 & 2 \\
\hline
\end{tabular}

Figure 2: Three pairwise orthogonal $4 \times 7$ Youden rectangles.

Given that we have found a triple of pairwise orthogonal Youden rectangles it is natural to ask for larger tuples. Aggarwal Agg75 proved that the upper bound $n-1$ holds for the number of mutually orthogonal Latin rectangles as well as for squares.

The sizes $(n-1) \times n$ are mostly not interesting in this regard since a set of $n-1$ mutually orthogonal Youden rectangles can be generated by removing one row from a set of $n-1$ MOLS. In particular, only the sizes $5 \times 6,3 \times 7$ and $4 \times 7$ are of interest for this analysis.

Observation 4.2. There exists a set of four pairwise orthogonal $5 \times 6$ Youden rectangles, a set of six pairwise orthogonal $3 \times 7$ Youden rectangles, and a set of six pairwise orthogonal $4 \times 7$ Youden rectangles.

Examples of such tuples are given in Appendix E For $n=7$ and $k=3,4$ this reaches the theoretical upper bound, so certainly these examples are maxima. For $n=6, k=5$ it is not ruled out immediately that there could exist a 5tuple of mutually orthogonal Youden rectangles, but using a simple specialized program, we have checked that this is not the case, and that the example we present is actually a maximum.

\subsection{Autotopism Groups of Triples}

Our next aim is to investigate the autotopism groups of the triples of MOLR. We present the order of the autotopism group for all triples of MOLR, and track the order of the autotopism group of those triples of rectangles which can be extended to triples of MOLS.

In Table 3 we give the maximum order of an autotopism group for size $k \times n$, the second largest order, and the number of non-isotopic triples which have groups with these cardinalities. Moreover, we also give the number of triples with trivial autotopism group. 


\begin{tabular}{|r|r|r|r|}
\hline \multirow{2}{*}{ Size } & \multirow{2}{*}{ Trivial group } & \multicolumn{2}{|c|}{$\begin{array}{c}\text { Number of autotopisms/ } \\
\text { Number of triples }\end{array}$} \\
\cline { 3 - 4 } & & $\begin{array}{c}\text { Second } \\
\text { maximum }\end{array}$ & \multicolumn{1}{c|}{ Maximum } \\
\hline $2 \times 4$ & 0 & $16 / 1$ & $48 / 1$ \\
\hline $3 \times 4$ & 0 & 0 & $72 / 1$ \\
\hline $4 \times 4$ & 0 & 0 & $288 / 1$ \\
\hline \hline $2 \times 5$ & 0 & $6 / 2$ & $10 / 1$ \\
\hline $3 \times 5$ & 0 & 0 & $10 / 1$ \\
\hline $4 \times 5$ & 0 & 0 & $20 / 1$ \\
\hline $5 \times 5$ & 0 & 0 & $100 / 1$ \\
\hline \hline $2 \times 6$ & 24 & $36 / 1$ & $72 / 1$ \\
\hline $3 \times 6$ & 1980 & $18 / 4$ & $36 / 4$ \\
\hline $4 \times 6$ & 93 & $24 / 1$ & $36 / 3$ \\
\hline $5 \times 6$ & 0 & $9 / 1$ & $18 / 2$ \\
\hline \hline $2 \times 7$ & 2300 & $14 / 3$ & $42 / 1$ \\
\hline $3 \times 7$ & 65822447 & $42 / 1$ & $63 / 1$ \\
\hline $4 \times 7$ & 323002195 & $42 / 1$ & $63 / 1$ \\
\hline $5 \times 7$ & 52981 & $21 / 1$ & $42 / 1$ \\
\hline $6 \times 7$ & 1 & $42 / 3$ & $126 / 1$ \\
\hline $7 \times 7$ & 0 & $294 / 3$ & $882 / 1$ \\
\hline
\end{tabular}

Table 3: The number of non-isotopic triples of MOLR with autotopism group of given orders.

We see that there are no triples of MOLR of order $n=4,5$ with trivial autotopism group. We also see that triples with maximum $k$, given the order $n$, namely $4 \times 4,5 \times 5,5 \times 6,7 \times 7$, always have a non-trivial autotopism group.

In Tables 4 5, 6, and 7 we give complete statistics on the number of triples with autotopism groups of given orders. We see here that for $n=6,7$ having a trivial autotopism group becomes the most common case, though not for each $k$ separately.

Here we also track the order of the autotopism group for the triples of MOLR of each order which can be extended to a triple of MOLS. For $n=6$ we follow the triple of MOLR which can be extended to a $5 \times 6$ triple of MOLR. The numbering of those cases correspond to the number given each triple of MOLS in the appendices, see Appendix A, B, C, D. Note that for orders 4 and 5 there is only one maximum example with respect to isotopism. 


\begin{tabular}{|c|c|c|}
\hline \multicolumn{3}{|c|}{$2 \times 4$} \\
\hline Group cardinality & 16 & 48 \\
\hline Number of triples & 1 & 1 \\
\hline MOLS case & & 1 \\
\hline \hline \multicolumn{2}{|c|}{$3 \times 4$} \\
\hline Group cardinality & 72 \\
\hline Number of triples & 1 \\
\hline MOLS case & 1 \\
\hline \hline \multicolumn{2}{|c|}{$4 \times 4$} \\
\hline Group cardinality & 288 \\
\hline Number of triples & 1 \\
\hline MOLS case & 1 \\
\hline
\end{tabular}

Table 4: Autotopism groups of triples of order 4 .

\begin{tabular}{|c|c|c|c|}
\hline \multicolumn{4}{|c|}{$2 \times 5$} \\
\hline Group cardinality & 2 & 6 & 10 \\
\hline Number of triples & 1 & 2 & 1 \\
\hline MOLS case & & & 1 \\
\hline \hline \multicolumn{2}{|c|}{$3 \times 5$} \\
\hline Group cardinality & & 10 \\
\hline Number of triples & & 1 \\
\hline MOLS case & \\
\hline \hline \multicolumn{3}{|c|}{$4 \times 5$} \\
\hline Group cardinality & \\
\hline Number of triples & \\
\hline MOLS case & \\
\hline \hline \multicolumn{2}{|c|}{$5 \times 5$} \\
\hline Group cardinality & \\
\hline Number of triples & 1 \\
\hline MOLS case & \\
\hline
\end{tabular}

Table 5: Autotopism groups of triples of order 5 . 


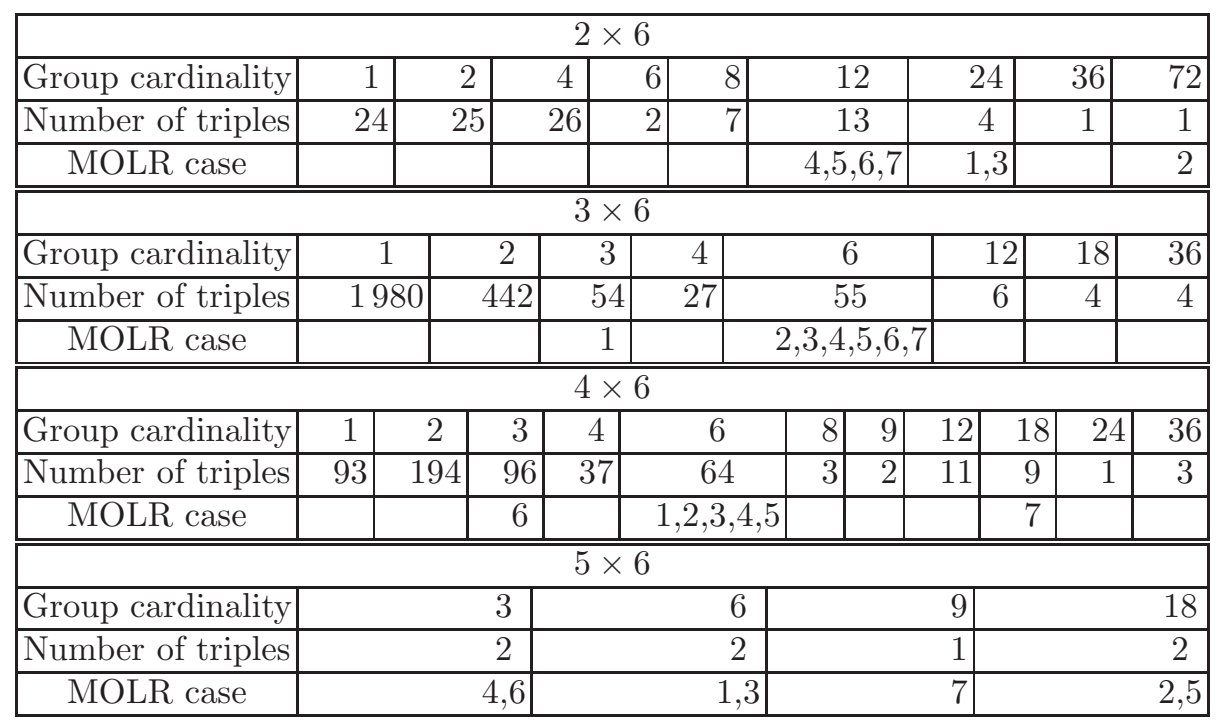

Table 6: Autotopism groups of triples of order 6 . 


\begin{tabular}{|c|c|c|c|c|c|c|c|c|c|c|c|c|}
\hline \multicolumn{13}{|c|}{$2 \times 7$} \\
\hline Group cardinality & 1 & 2 & $3 \mid$ & 4 & & 6 & & 12 & & 14 & & 42 \\
\hline Number of triples & 2300 & 512 & 3 & 28 & & 9 & & $\overline{2}$ & & 3 & & 1 \\
\hline MOLS case & & & & & & & & & & $1,2,4$ & & 3 \\
\hline \multicolumn{13}{|c|}{$3 \times 7$} \\
\hline Group cardinality & 1 & 2 & 3 & 6 & 7 & 9 & 14 & & 18 & 21 & 42 & 63 \\
\hline Number of triples & 6582244 & 60195 & 635 & 143 & \begin{tabular}{|l|l|}
17 \\
\end{tabular} & 3 & 3 & & 4 & 4 & 1 & 1 \\
\hline MOLS case & & & & & & & 1,2, & & & & 3 & \\
\hline \multicolumn{13}{|c|}{$4 \overline{4 \times 7}$} \\
\hline Group cardinality & 1 & 2 & 3 & 4 & 6 & \begin{tabular}{|l|l}
7 & $!$ \\
\end{tabular} & \begin{tabular}{|l|l|}
9 & 12 \\
\end{tabular} & 214 & 18 & 21 & 42 & \\
\hline Number of triples & 32300219 & 107997 & 1975 & \begin{tabular}{|l|l|l|}
120 & 1 \\
\end{tabular} & 116 & \begin{tabular}{|l|l|}
43 & 1 \\
\end{tabular} & \begin{tabular}{l|l|}
10 & 3 \\
\end{tabular} & 8 & 2 & 6 & 1 & 1 \\
\hline MOLS case & & & & & & & & & & 1,2, & & 3 \\
\hline \multicolumn{13}{|c|}{$5 \times 7$} \\
\hline Group cardinality & 1 & 2 & \begin{tabular}{l|l}
3 & \\
\end{tabular} & 4 & 6 & & 7 & 1 & $\overline{14}$ & 2 & 1 & 42 \\
\hline Number of triples & 52981 & 2500 & 32 & 5 & 2 & & 15 & $\varepsilon$ & 8 & 1 & 1 & 1 \\
\hline MOLS case & & & & & & & & & 2,4 & & & 3 \\
\hline \multicolumn{13}{|c|}{$6 \times 7$} \\
\hline Group cardinality & 1 & 2 & 3 & 4 & 6 & & 12 & & & 42 & & 126 \\
\hline Number of triples & 1 & 4 & 1 & 1 & 3 & & 2 & & & 3 & & 1 \\
\hline MOLS case & & & & & & & $\overline{1,2,4}$ & & & & & 3 \\
\hline \multicolumn{13}{|c|}{$\overline{7 \times 7}$} \\
\hline Group cardinality & & & & 294 & & & & & & & & 882 \\
\hline Number of triples & & & & 3 & & & & & & & & 1 \\
\hline MOLS case & & & & $\overline{1,2,4}$ & & & & & & & & 3 \\
\hline
\end{tabular}

Table 7: Autotopism groups of triples of order 7 .

We make the quite striking observation that for $n \leq 7$ the triples of MOLR which can be extended to a triple of MOLS, or MOLR with maximal $k$ for $n=6$, always have a non-trivial autotopism group. Given the paucity of such triples for larger $n$ it would be interesting if this non-triviality can be proven to hold for all $n \geq 8$ as well, or if there are non-symmetrical examples as well, especially in view of the result of MMM07 that in any triple of MOLS for $n=10$, each of the three Latin squares involved must have a trivial symmetry group.

\section{$5 \quad$ Larger orders}

Our methods and program can be applied to larger values of $n$ as well, but here the number of triples of MOLR quickly becomes unmanageable. It is easy to generate all non-isotopic $2 \times 8$ triples, and we found that the number of these is:

$$
2 \times 8: \quad 188126
$$


Going to $3 \times 8$ becomes significantly harder, but we have done this step as well. Using the parallel machine this took approximately 150000 core hours, and saving the full output would have required several terabytes of disk space. We found that the number of non-isotopic $3 \times 8$ triples of MOLR is:

$$
3 \times 8: \quad 3321281937279
$$

Given that we expect the number of $4 \times 8$ triples to be significantly larger, we conclude that at this point it is not possible to do a full enumeration for $n \geq 8$ while saving the resulting triples to disk.

However, in Subsection 4.3 we noted that for $n \leq 7$ the $k \times n$ triples of MOLR with maximum $k$ all have non-trivial autotopism group, and that they can be constructed by extending triples with lower $k$ and non-trivial autotopisms. With this in mind we recursively define a triple of MOLR to be stepwise symmetric if it has non-trivial autotopism group and is an extension of a stepwise symmetric triple of MOLR. We can enumerate stepwise symmetric triples of MOLR by starting with the unique $1 \times n$ normalized triple and after each extension step removing the triples with trivial autotopism group. In Table 8 we record the number of stepwise symmetric triples of MOLR for $n=8$. As we can see, the total number of non-isotopic $3 \times 8$ triples is vastly larger than the number of stepwise symmetric such triples. As expected, we find that the stepwise symmetric class includes examples of triples of $8 \times 8$ MOLS. We also note that there are such triples of squares that are not part of the projective plane of order 8 .

\begin{tabular}{|r|r|rr|}
\hline \multirow{2}{*}{ Size } & \multicolumn{3}{|c|}{ non-isotopic } \\
\cline { 2 - 4 } & \multicolumn{1}{|c|}{ All } & \multicolumn{2}{|c|}{ Youden } \\
\hline $2 \times 8$ & 10211 & \multicolumn{2}{|c|}{0} \\
\hline $3 \times 8$ & 22747116 & & 0 \\
\hline $4 \times 8$ & 3796573635 & $\lambda_{c c}^{p}=1$ & 26747355 \\
\hline $5 \times 8$ & 2503469320 & $\lambda_{c c}^{p}=2$ & 2503469320 \\
\hline $6 \times 8$ & 5572534 & $\lambda_{c c}^{p}=4$ & 5572534 \\
\hline $7 \times 8$ & 751 & $\lambda_{c c}=6$ & 751 \\
\hline $8 \times 8$ & 72 & $\lambda_{c c}=8$ & 72 \\
\hline
\end{tabular}

Table 8: The number of stepwise symmetric triples of MOLR for $n=8$.

For $k=7,8$ the $k \times n$ rectangles are automatically Youden rectangles. For $k=4$, the bounds on $\lambda_{c c}^{p}$ discussed in Subsection 4.2 leave $\lambda_{c c}^{p}=1$ as a possible non-zero value, and for $k=5$ and $k=6$, we must have $2 \leq \lambda_{c c}^{p} \leq \frac{20}{7}$ and $4 \leq \lambda_{c c}^{p} \leq \frac{30}{7}$ respectively, leaving only the possible values $\lambda_{c c}^{p}=2$ and $\lambda_{c c}^{p}=4$.

We have also checked the 72 triples of MOLS for maximality with respect to adding further squares, and we find that 70 of the triples are maximal. One of the two remaining triples extends to a maximal 4-tuple of MOLS, and the other extends to a full 7-tuple of MOLS, corresponding to the projective plane of this order. 
As for smaller $n$, for $n=8$ we have also investigated the order of the autotopism groups for the stepwise symmetric triples of MOLR. The results are shown in Table 9. Here we note that for each $k \leq 7$ the most common group order is 2 , and similarly to the non-symmetric case there are no $8 \times 8$ triples with an autotopism group of the smallest possible order, namely 2 . We also note that order 4 is more common than order 3 , so the number of triples is not decreasing in the order of the autotopism group.

One noteworthy feature is the number of rows in the triples with the maximum autotopism group order. For $n \leq 7$, with the exception of $n=6$, this has been achieved by triples with the maximum possible $k$, but when $n=8$, we instead find the maximum among the $4 \times 8$ triples, where we find two triples with autotopism group of order $2304=2^{8} \cdot 3^{2}$. These triples are presented in Figure 3. Both of these triples are maximal with respect to addition of more rows.

\begin{tabular}{|l|l|l|l||l|l|l|l|l|l|l|l||l|l|l|l|l|l|l|l||l|l|l|l|}
\hline 0 & 1 & 2 & 3 & 4 & 5 & 6 & 7 \\
\hline 3 & 2 & 1 & 0 & 7 & 6 & 5 & 4 \\
\hline 2 & 3 & 0 & 1 & 6 & 7 & 4 & 5 \\
\hline 1 & 0 & 3 & 2 & 5 & 4 & 7 & 6 \\
\hline 0 & 1 & 2 & 3 & 4 & 5 & 6 & 7 \\
\hline 2 & 3 & 0 & 1 & 6 & 7 & 4 & 5 \\
\hline 1 & 0 & 3 & 2 & 5 & 4 & 7 & 6 \\
\hline 3 & 2 & 1 & 0 & 7 & 6 & 5 & 4 \\
\hline
\end{tabular}$\quad$\begin{tabular}{|l|l|l|l|l|l|l|l|}
0 & 1 & 2 & 3 & 4 & 5 & 6 & 7 \\
\hline 1 & 0 & 3 & 2 & 5 & 4 & 7 & 6 \\
\hline 3 & 2 & 1 & 0 & 7 & 6 & 5 & 4 \\
\hline 2 & 3 & 0 & 1 & 6 & 7 & 4 & 5 \\
\hline
\end{tabular}

(a) First triple of mutually orthogonal $8 \times 4$ MOLR.

\begin{tabular}{|c|c|c|c|c|c|c|c|c|c|c|c|c|c|c|c|c|c|c|c|c|c|c|}
\hline 0 & 1 & 2 & & 4 & 5 & & & \begin{tabular}{|l|}
0 \\
\end{tabular} & \begin{tabular}{|l|}
1 \\
\end{tabular} & 2 & 3 & 4 & 5 & \begin{tabular}{l|l}
6 & 7
\end{tabular} & \begin{tabular}{|l|}
0 \\
\end{tabular} & 1 & 2 & \begin{tabular}{|l|}
3 \\
\end{tabular} & \begin{tabular}{|l|}
4 \\
\end{tabular} & \begin{tabular}{|l|}
5 \\
\end{tabular} & & \\
\hline 3 & 2 & \begin{tabular}{l|l}
1 & 0
\end{tabular} & $\overline{0}$ & 7 & 6 & 5 & 4 & \begin{tabular}{|l|}
2 \\
\end{tabular} & 3 & 0 & 1 & 6 & 7 & \begin{tabular}{l|l}
4 \\
\end{tabular} & \begin{tabular}{|l|}
1 \\
\end{tabular} & 0 & 3 & 2 & 5 & 4 & 7 & 6 \\
\hline 2 & 3 & $\overline{0}$ & $\overline{1}$ & 5 & \begin{tabular}{|l|}
4 \\
\end{tabular} & & 6 & \begin{tabular}{|l|}
1 \\
\end{tabular} & \begin{tabular}{|l|l|}
0 \\
\end{tabular} & 3 & 2 & 7 & 6 & \begin{tabular}{l|l}
5 & 4
\end{tabular} & \begin{tabular}{|l|}
3 \\
\end{tabular} & 2 & 1 & 0 & 6 & 7 & $\overline{4}$ & 5 \\
\hline \begin{tabular}{|l|}
1 \\
\end{tabular} & 0 & 3 & 2 & 6 & \begin{tabular}{|l|}
7 \\
\end{tabular} & & 5 & \begin{tabular}{|l|}
3 \\
\end{tabular} & 2 & 1 & 0 & 5 & 4 & \begin{tabular}{l|l}
7 & 6 \\
\end{tabular} & \begin{tabular}{|l|}
2 \\
\end{tabular} & 3 & 0 & 1 & 7 & 6 & & 4 \\
\hline
\end{tabular}

(b) Second triple of mutually orthogonal $8 \times 4$ MOLR.

Figure 3: The two $4 \times 8$ triples with autotopism group of order 2304 .

As pointed out to us by Rosemary Bailey these examples can be generated from cosets of the elementary Abelian group of order 32 (see [Bai96]), or even simpler, by juxtaposing two triples of MOLS of order 4. The double line in the above examples indicates this juxtaposition, and we have chosen representatives from the isotopism classes of triples of $4 \times 4$ MOLS so that the left side of both the above examples of triples of $4 \times 8$ MOLR corresponds exactly to our chosen representative of the isotopic triples of MOLS of order 4. In Figure 3a the right side also corresponds exactly to the same triple of $4 \times 4$ MOLS, by renaming the symbols $4,5,6,7$ to $0,1,2,3$, respectively. In Figure 3b, rows 3 and 4 in the $4 \times 4$ MOLS on the right side are switched.

Based on this observation, we formulate the following result, which follows immediately from juxtaposing the two $t$-tuples of MOLR, and introducing new symbol names for the triple of MOLR on the right side.

Proposition 5.1. If there exists a $t$-tuple of $k \times n_{1} M O L R$, and a $t$-tuple of $k \times n_{2} M O L R$, then there exists a $t$-tuple of $k \times\left(n_{1}+n_{2}\right)$ MOLR. 


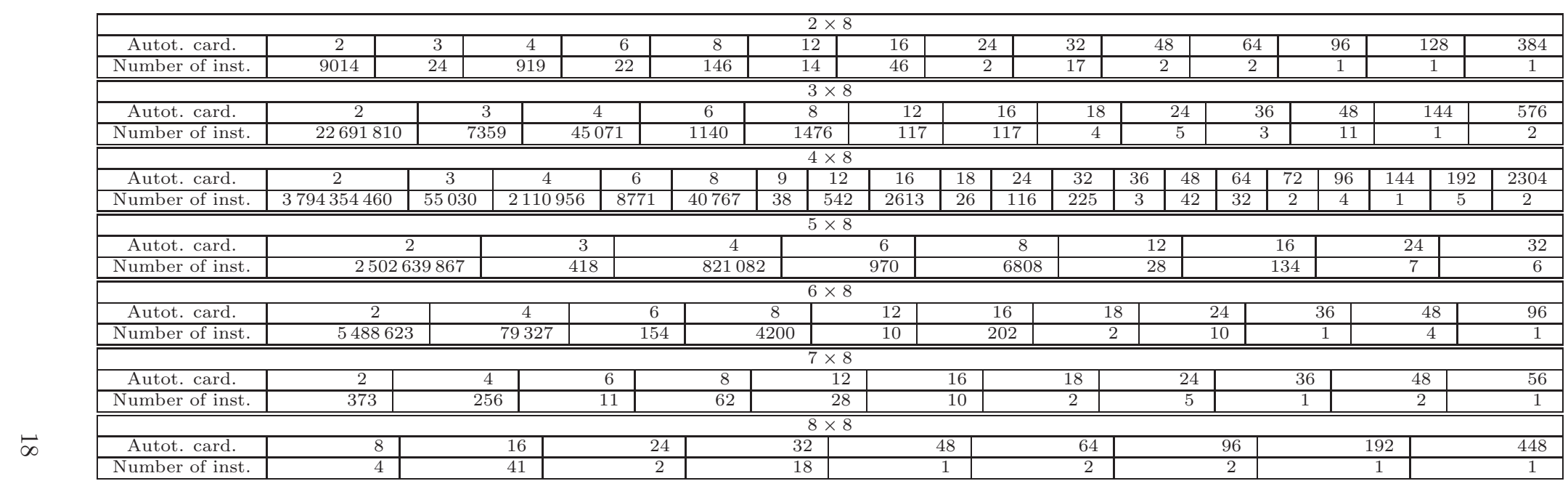

Table 9: The order of the autotopism group for the stepwise symmetric triples of $k \times 8$ rectangles. 
For example, there exists a 4 -tuple of $5 \times 10$ MOLR, since there exists a 4 -tuple of $5 \times 5$ MOLR. However, the triples of MOLR formed by applying Proposition 5.1 will clearly have poor properties regarding adding more rows.

\section{Acknowledgments}

The computational work was performed on resources provided by the Swedish National Infrastructure for Computing (SNIC) at High Performance Computing Center North (HPC2N). This work was supported by the Swedish strategic research programme eSSENCE. This work was supported by The Swedish Research Council grant 2014-4897.

\section{References}

[Agg75] K. R. Aggarwal, Mutually partially orthogonal Latin rectangles and mutually partially orthogonal Latin cubes of second order, Proc. Indian Nat. Sci. Acad. Part A 41 (1975), no. 3, 216-223. MR 0543054

[Bai96] R. A. Bailey, Orthogonal partitions in designed experiments, Des. Codes Cryptogr. 8 (1996), no. 1-2, 45-77, Special issue dedicated to Hanfried Lenz. MR 1393974

$\left[\mathrm{BKL}^{+} 17\right]$ B. Barber, D. Kühn, A. Lo, D. Osthus, and A. Taylor, Clique decompositions of multipartite graphs and completion of Latin squares, J. Combin. Theory Ser. A 151 (2017), 146-201. MR 3663493

[Bos38] R. C. Bose, On the application of the properties of Galois fields to the problem of construction of hyper-Graeco-Latin squares, Sankhya: The Indian Journal of Statistics (1933-1960) 3 (1938), no. 4, 323-338.

[BSP60] R. C. Bose, S. S. Shrikhande, and E. T. Parker, Further results on the construction of mutually orthogonal Latin squares and the falsity of Euler's conjecture, Canad. J. Math. 12 (1960), 189-203. MR 0122729

[CES60] S. Chowla, P. Erdős, and E. G. Straus, On the maximal number of pairwise orthogonal Latin squares of a given order, Canad. J. Math. 12 (1960), 204-208. MR 0122730

[Cla63] G. M. Clarke, A second set of treatments in a Youden square design, Biometrics 19 (1963), 98-104. MR 0175258

[Cla67] G. M. Clarke, Four-way balanced designs based on Youden squares with 5, 6, or 7 treatments, Biometrics 23 (1967), no. 4, 803-812. MR 0220409

[EW16] J. Egan and I. M. Wanless, Enumeration of MOLS of small order, Math. Comp. 85 (2016), no. 298, 799-824. MR 3434882 
[HK08] K. Hinkelmann and O. Kempthorne, Design and analysis of experiments. Vol. 1, second ed., Wiley Series in Probability and Statistics, Wiley-Interscience [John Wiley \& Sons], Hoboken, NJ, 2008, Introduction to experimental design. MR 2363107

[Lam91] C. W. H. Lam, The search for a finite projective plane of order 10, Amer. Math. Monthly 98 (1991), no. 4, 305-318. MR 1103185

[LM98] Charles F. Laywine and Gary L. Mullen, Discrete mathematics using Latin squares, Wiley-Interscience Series in Discrete Mathematics and Optimization, John Wiley \& Sons, Inc., New York, 1998, A WileyInterscience Publication. MR 1644242

[LTS89] C. W. H. Lam, L. Thiel, and S. Swiercz, The nonexistence of finite projective planes of order 10, Canad. J. Math. 41 (1989), no. 6, 1117-1123. MR 1018454

[MMM07] B. D. McKay, A. Meynert, and W. Myrvold, Small Latin squares, quasigroups, and loops, J. Combin. Des. 15 (2007), no. 2, 98-119. MR 2291523

[PM17] D. A. Preece and J. P. Morgan, Multi-layered Youden rectangles, J. Combin. Des. 25 (2017), no. 2, 75-84. MR 3592324

[Pre66] D. A. Preece, Classifying Youden rectangles, Journal of the Royal Statistical Society. Series B (Methodological) 28 (1966), no. 1, 118130 .

[PVP99] D. A. Preece, B. J. Vowden, and N. C. K. Phillips, Double Youden rectangles of sizes $p \times(2 p+1)$ and $(p+1) \times(2 p+1)$, Ars Combin. 51 (1999), 161-171. MR 1675144

[Tar00] G. Tarry, Le probleme de 36 officiers, Canad. J. Math. 1,2 (1900), 122-123, 170-203.

[Tod85] D. T. Todorov, Three mutually orthogonal Latin squares of order 14, Ars Combin. 20 (1985), 45-47. MR 824847

[Web] t-tuples of orthogonal Latin rectangles, http://abel.math.umu.se/ klasm/Data/ortlatin/.

[You37] W. J. Youden, Use of incomplete block replications in estimating tobacco-mosaic virus, Contributions from the Boyce Thompson Institute (1937), no. 9, 41-48. 
A The unique (up to isotopism) triple of $4 \times 4$ MOLS

\begin{tabular}{|l|l|l|l|}
\hline 0 & 1 & 2 & 3 \\
\hline 3 & 2 & 1 & 0 \\
\hline 2 & 3 & 0 & 1 \\
\hline 1 & 0 & 3 & 2 \\
\hline
\end{tabular}

\begin{tabular}{|l|l|l|l|}
\hline 0 & 1 & 2 & 3 \\
\hline 2 & 3 & 0 & 1 \\
\hline 1 & 0 & 3 & 2 \\
\hline 3 & 2 & 1 & 0 \\
\hline
\end{tabular}

\begin{tabular}{|l|l|l|l|}
\hline 0 & 1 & 2 & 3 \\
\hline 1 & 0 & 3 & 2 \\
\hline 3 & 2 & 1 & 0 \\
\hline 2 & 3 & 0 & 1 \\
\hline
\end{tabular}

B The unique (up to isotopism) triple of $5 \times 5$ MOLS

\begin{tabular}{|l|l|l|l|l|}
\hline 0 & 1 & 2 & 3 & 4 \\
\hline 4 & 3 & 1 & 0 & 2 \\
\hline 3 & 2 & 4 & 1 & 0 \\
\hline 2 & 0 & 3 & 4 & 1 \\
\hline 1 & 4 & 0 & 2 & 3 \\
\hline
\end{tabular}

\begin{tabular}{|l|l|l|l|l|}
\hline 0 & 1 & 2 & 3 & 4 \\
\hline 3 & 2 & 4 & 1 & 0 \\
\hline 4 & 3 & 1 & 0 & 2 \\
\hline 1 & 4 & 0 & 2 & 3 \\
\hline 2 & 0 & 3 & 4 & 1 \\
\hline
\end{tabular}

\begin{tabular}{|l|l|l|l|l|}
\hline 0 & 1 & 2 & 3 & 4 \\
\hline 2 & 0 & 3 & 4 & 1 \\
\hline 1 & 4 & 0 & 2 & 3 \\
\hline 3 & 2 & 4 & 1 & 0 \\
\hline 4 & 3 & 1 & 0 & 2 \\
\hline
\end{tabular}

C The seven non-isotopic triples of $5 \times 6$ MOLR

1.

\begin{tabular}{|l|l|l|l|l|l|}
\hline 0 & 1 & 2 & 3 & 4 & 5 \\
\hline 5 & 4 & 3 & 2 & 1 & 0 \\
\hline 4 & 5 & 1 & 0 & 3 & 2 \\
\hline 3 & 2 & 5 & 4 & 0 & 1 \\
\hline 2 & 0 & 4 & 1 & 5 & 3 \\
\hline
\end{tabular}

\begin{tabular}{|l|l|l|l|l|l|}
\hline 0 & 1 & 2 & 3 & 4 & 5 \\
\hline 4 & 5 & 1 & 0 & 3 & 2 \\
\hline 3 & 2 & 5 & 4 & 0 & 1 \\
\hline 2 & 4 & 3 & 1 & 5 & 0 \\
\hline 5 & 3 & 0 & 2 & 1 & 4 \\
\hline
\end{tabular}

\begin{tabular}{|l|l|l|l|l|l|}
\hline 0 & 1 & 2 & 3 & 4 & 5 \\
\hline 3 & 2 & 5 & 4 & 0 & 1 \\
\hline 5 & 0 & 4 & 2 & 1 & 3 \\
\hline 4 & 5 & 1 & 0 & 3 & 2 \\
\hline 1 & 4 & 3 & 5 & 2 & 0 \\
\hline
\end{tabular}

2.

\begin{tabular}{|l|l|l|l|l|l|}
\hline 0 & 1 & 2 & 3 & 4 & 5 \\
\hline 5 & 4 & 3 & 2 & 1 & 0 \\
\hline 4 & 3 & 5 & 1 & 0 & 2 \\
\hline 3 & 5 & 4 & 0 & 2 & 1 \\
\hline 2 & 0 & 1 & 4 & 5 & 3 \\
\hline
\end{tabular}

\begin{tabular}{|l|l|l|l|l|l|}
\hline 0 & 1 & 2 & 3 & 4 & 5 \\
\hline 4 & 3 & 5 & 1 & 0 & 2 \\
\hline 5 & 4 & 3 & 2 & 1 & 0 \\
\hline 2 & 0 & 1 & 4 & 5 & 3 \\
\hline 3 & 5 & 4 & 0 & 2 & 1 \\
\hline
\end{tabular}

\begin{tabular}{|l|l|l|l|l|l|}
\hline 0 & 1 & 2 & 3 & 4 & 5 \\
\hline 3 & 5 & 4 & 0 & 2 & 1 \\
\hline 2 & 0 & 1 & 4 & 5 & 3 \\
\hline 5 & 4 & 3 & 2 & 1 & 0 \\
\hline 4 & 3 & 5 & 1 & 0 & 2 \\
\hline
\end{tabular}


3.

\begin{tabular}{|l|l|l|l|l|l|}
\hline 0 & 1 & 2 & 3 & 4 & 5 \\
\hline 5 & 4 & 3 & 2 & 1 & 0 \\
\hline 4 & 3 & 5 & 0 & 2 & 1 \\
\hline 3 & 5 & 4 & 1 & 0 & 2 \\
\hline 1 & 2 & 0 & 5 & 3 & 4 \\
\hline
\end{tabular}

\begin{tabular}{|l|l|l|l|l|l|}
\hline 0 & 1 & 2 & 3 & 4 & 5 \\
\hline 4 & 3 & 5 & 0 & 2 & 1 \\
\hline 5 & 4 & 3 & 2 & 1 & 0 \\
\hline 2 & 0 & 1 & 4 & 5 & 3 \\
\hline 3 & 5 & 4 & 1 & 0 & 2 \\
\hline
\end{tabular}

\begin{tabular}{|l|l|l|l|l|l|}
\hline 0 & 1 & 2 & 3 & 4 & 5 \\
\hline 3 & 5 & 4 & 1 & 0 & 2 \\
\hline 2 & 0 & 1 & 4 & 5 & 3 \\
\hline 5 & 4 & 3 & 2 & 1 & 0 \\
\hline 4 & 3 & 5 & 0 & 2 & 1 \\
\hline
\end{tabular}

4.

\begin{tabular}{|l|l|l|l|l|l|}
\hline 0 & 1 & 2 & 3 & 4 & 5 \\
\hline 5 & 4 & 3 & 1 & 0 & 2 \\
\hline 4 & 3 & 5 & 2 & 1 & 0 \\
\hline 2 & 0 & 1 & 5 & 3 & 4 \\
\hline 1 & 2 & 0 & 4 & 5 & 3 \\
\hline
\end{tabular}

\begin{tabular}{|l|l|l|l|l|l|}
\hline 0 & 1 & 2 & 3 & 4 & 5 \\
\hline 4 & 3 & 5 & 2 & 1 & 0 \\
\hline 1 & 2 & 0 & 5 & 3 & 4 \\
\hline 3 & 5 & 4 & 1 & 0 & 2 \\
\hline 5 & 4 & 3 & 0 & 2 & 1 \\
\hline
\end{tabular}

\begin{tabular}{|l|l|l|l|l|l|}
\hline 0 & 1 & 2 & 3 & 4 & 5 \\
\hline 2 & 0 & 1 & 5 & 3 & 4 \\
\hline 5 & 4 & 3 & 0 & 2 & 1 \\
\hline 1 & 2 & 0 & 4 & 5 & 3 \\
\hline 4 & 3 & 5 & 2 & 1 & 0 \\
\hline
\end{tabular}

5.

\begin{tabular}{|l|l|l|l|l|l|}
\hline 0 & 1 & 2 & 3 & 4 & 5 \\
\hline 5 & 4 & 3 & 1 & 0 & 2 \\
\hline 4 & 3 & 5 & 2 & 1 & 0 \\
\hline 2 & 0 & 1 & 5 & 3 & 4 \\
\hline 1 & 2 & 0 & 4 & 5 & 3 \\
\hline
\end{tabular}

\begin{tabular}{|l|l|l|l|l|l|}
\hline 0 & 1 & 2 & 3 & 4 & 5 \\
\hline 4 & 3 & 5 & 2 & 1 & 0 \\
\hline 1 & 2 & 0 & 5 & 3 & 4 \\
\hline 3 & 5 & 4 & 1 & 0 & 2 \\
\hline 5 & 4 & 3 & 0 & 2 & 1 \\
\hline
\end{tabular}

\begin{tabular}{|l|l|l|l|l|l|}
\hline 0 & 1 & 2 & 3 & 4 & 5 \\
\hline 2 & 0 & 1 & 5 & 3 & 4 \\
\hline 5 & 4 & 3 & 0 & 2 & 1 \\
\hline 1 & 2 & 0 & 4 & 5 & 3 \\
\hline 3 & 5 & 4 & 2 & 1 & 0 \\
\hline
\end{tabular}

6.

\begin{tabular}{|l|l|l|l|l|l|}
\hline 0 & 1 & 2 & 3 & 4 & 5 \\
\hline 5 & 4 & 3 & 1 & 0 & 2 \\
\hline 4 & 3 & 5 & 0 & 2 & 1 \\
\hline 3 & 5 & 4 & 2 & 1 & 0 \\
\hline 1 & 2 & 0 & 4 & 5 & 3 \\
\hline
\end{tabular}

\begin{tabular}{|l|l|l|l|l|l|}
\hline 0 & 1 & 2 & 3 & 4 & 5 \\
\hline 4 & 3 & 5 & 2 & 1 & 0 \\
\hline 2 & 0 & 1 & 5 & 3 & 4 \\
\hline 1 & 2 & 0 & 4 & 5 & 3 \\
\hline 3 & 5 & 4 & 1 & 0 & 2 \\
\hline
\end{tabular}

\begin{tabular}{|l|l|l|l|l|l|}
\hline 0 & 1 & 2 & 3 & 4 & 5 \\
\hline 2 & 0 & 1 & 5 & 3 & 4 \\
\hline 1 & 2 & 0 & 4 & 5 & 3 \\
\hline 5 & 4 & 3 & 1 & 0 & 2 \\
\hline 4 & 3 & 5 & 2 & 1 & 0 \\
\hline
\end{tabular}

7.

\begin{tabular}{|l|l|l|l|l|l|}
\hline 0 & 1 & 2 & 3 & 4 & 5 \\
\hline 5 & 4 & 3 & 1 & 0 & 2 \\
\hline 4 & 3 & 5 & 0 & 2 & 1 \\
\hline 3 & 5 & 4 & 2 & 1 & 0 \\
\hline 2 & 0 & 1 & 5 & 3 & 4 \\
\hline
\end{tabular}

\begin{tabular}{|l|l|l|l|l|l|}
\hline 0 & 1 & 2 & 3 & 4 & 5 \\
\hline 4 & 3 & 5 & 2 & 1 & 0 \\
\hline 2 & 0 & 1 & 5 & 3 & 4 \\
\hline 1 & 2 & 0 & 4 & 5 & 3 \\
\hline 5 & 4 & 3 & 0 & 2 & 1 \\
\hline
\end{tabular}

\begin{tabular}{|l|l|l|l|l|l|}
\hline 0 & 1 & 2 & 3 & 4 & 5 \\
\hline 2 & 0 & 1 & 5 & 3 & 4 \\
\hline 1 & 2 & 0 & 4 & 5 & 3 \\
\hline 5 & 4 & 3 & 0 & 2 & 1 \\
\hline 3 & 5 & 4 & 1 & 0 & 2 \\
\hline
\end{tabular}




\section{The four non-isotopic triples of $7 \times 7$ MOLS}

1.

\begin{tabular}{|l|l|l|l|l|l|l|}
\hline 0 & 1 & 2 & 3 & 4 & 5 & 6 \\
\hline 6 & 5 & 4 & 2 & 1 & 0 & 3 \\
\hline 5 & 4 & 3 & 6 & 2 & 1 & 0 \\
\hline 4 & 3 & 0 & 5 & 6 & 2 & 1 \\
\hline 3 & 0 & 1 & 4 & 5 & 6 & 2 \\
\hline 2 & 6 & 5 & 1 & 0 & 3 & 4 \\
\hline 1 & 2 & 6 & 0 & 3 & 4 & 5 \\
\hline
\end{tabular}

\begin{tabular}{|l|l|l|l|l|l|l|}
\hline 0 & 1 & 2 & 3 & 4 & 5 & 6 \\
\hline 5 & 4 & 3 & 6 & 2 & 1 & 0 \\
\hline 6 & 5 & 4 & 2 & 1 & 0 & 3 \\
\hline 2 & 6 & 5 & 1 & 0 & 3 & 4 \\
\hline 1 & 2 & 6 & 0 & 3 & 4 & 5 \\
\hline 4 & 3 & 0 & 5 & 6 & 2 & 1 \\
\hline 3 & 0 & 1 & 4 & 5 & 6 & 2 \\
\hline
\end{tabular}

\begin{tabular}{|l|l|l|l|l|l|l|}
\hline 0 & 1 & 2 & 3 & 4 & 5 & 6 \\
\hline 4 & 3 & 0 & 5 & 6 & 2 & 1 \\
\hline 2 & 6 & 5 & 1 & 0 & 3 & 4 \\
\hline 3 & 0 & 1 & 4 & 5 & 6 & 2 \\
\hline 6 & 5 & 4 & 2 & 1 & 0 & 3 \\
\hline 1 & 2 & 6 & 0 & 3 & 4 & 5 \\
\hline 5 & 4 & 3 & 6 & 2 & 1 & 0 \\
\hline
\end{tabular}

2.

\begin{tabular}{|l|l|l|l|l|l|l|}
\hline 0 & 1 & 2 & 3 & 4 & 5 & 6 \\
\hline 6 & 5 & 4 & 2 & 1 & 0 & 3 \\
\hline 5 & 4 & 3 & 6 & 2 & 1 & 0 \\
\hline 4 & 3 & 0 & 5 & 6 & 2 & 1 \\
\hline 3 & 0 & 1 & 4 & 5 & 6 & 2 \\
\hline 2 & 6 & 5 & 1 & 0 & 3 & 4 \\
\hline 1 & 2 & 6 & 0 & 3 & 4 & 5 \\
\hline
\end{tabular}

\begin{tabular}{|l|l|l|l|l|l|l|}
\hline 0 & 1 & 2 & 3 & 4 & 5 & 6 \\
\hline 5 & 4 & 3 & 6 & 2 & 1 & 0 \\
\hline 6 & 5 & 4 & 2 & 1 & 0 & 3 \\
\hline 2 & 6 & 5 & 1 & 0 & 3 & 4 \\
\hline 1 & 2 & 6 & 0 & 3 & 4 & 5 \\
\hline 4 & 3 & 0 & 5 & 6 & 2 & 1 \\
\hline 3 & 0 & 1 & 4 & 5 & 6 & 2 \\
\hline
\end{tabular}

\begin{tabular}{|l|l|l|l|l|l|l|}
\hline 0 & 1 & 2 & 3 & 4 & 5 & 6 \\
\hline 3 & 0 & 1 & 4 & 5 & 6 & 2 \\
\hline 1 & 2 & 6 & 0 & 3 & 4 & 5 \\
\hline 6 & 5 & 4 & 2 & 1 & 0 & 3 \\
\hline 4 & 3 & 0 & 5 & 6 & 2 & 1 \\
\hline 5 & 4 & 3 & 6 & 2 & 1 & 0 \\
\hline 2 & 6 & 5 & 1 & 0 & 3 & 4 \\
\hline
\end{tabular}

3.

\begin{tabular}{|l|l|l|l|l|l|l|}
\hline 0 & 1 & 2 & 3 & 4 & 5 & 6 \\
\hline 6 & 5 & 4 & 2 & 1 & 0 & 3 \\
\hline 5 & 4 & 3 & 6 & 2 & 1 & 0 \\
\hline 4 & 3 & 0 & 5 & 6 & 2 & 1 \\
\hline 3 & 0 & 1 & 4 & 5 & 6 & 2 \\
\hline 2 & 6 & 5 & 1 & 0 & 3 & 4 \\
\hline 1 & 2 & 6 & 0 & 3 & 4 & 5 \\
\hline
\end{tabular}

\begin{tabular}{|l|l|l|l|l|l|l|}
\hline 0 & 1 & 2 & 3 & 4 & 5 & 6 \\
\hline 4 & 3 & 0 & 5 & 6 & 2 & 1 \\
\hline 2 & 6 & 5 & 1 & 0 & 3 & 4 \\
\hline 3 & 0 & 1 & 4 & 5 & 6 & 2 \\
\hline 6 & 5 & 4 & 2 & 1 & 0 & 3 \\
\hline 1 & 2 & 6 & 0 & 3 & 4 & 5 \\
\hline 5 & 4 & 3 & 6 & 2 & 1 & 0 \\
\hline
\end{tabular}

\begin{tabular}{|l|l|l|l|l|l|l|}
\hline 0 & 1 & 2 & 3 & 4 & 5 & 6 \\
\hline 3 & 0 & 1 & 4 & 5 & 6 & 2 \\
\hline 1 & 2 & 6 & 0 & 3 & 4 & 5 \\
\hline 6 & 5 & 4 & 2 & 1 & 0 & 3 \\
\hline 4 & 3 & 0 & 5 & 6 & 2 & 1 \\
\hline 5 & 4 & 3 & 6 & 2 & 1 & 0 \\
\hline 2 & 6 & 5 & 1 & 0 & 3 & 4 \\
\hline
\end{tabular}

4.

\begin{tabular}{|l|l|l|l|l|l|l|}
\hline 0 & 1 & 2 & 3 & 4 & 5 & 6 \\
\hline 6 & 5 & 4 & 2 & 1 & 0 & 3 \\
\hline 5 & 4 & 3 & 6 & 2 & 1 & 0 \\
\hline 4 & 3 & 0 & 5 & 6 & 2 & 1 \\
\hline 3 & 0 & 1 & 4 & 5 & 6 & 2 \\
\hline 2 & 6 & 5 & 1 & 0 & 3 & 4 \\
\hline 1 & 2 & 6 & 0 & 3 & 4 & 5 \\
\hline
\end{tabular}

\begin{tabular}{|l|l|l|l|l|l|l|}
\hline 0 & 1 & 2 & 3 & 4 & 5 & 6 \\
\hline 4 & 3 & 0 & 5 & 6 & 2 & 1 \\
\hline 2 & 6 & 5 & 1 & 0 & 3 & 4 \\
\hline 3 & 0 & 1 & 4 & 5 & 6 & 2 \\
\hline 6 & 5 & 4 & 2 & 1 & 0 & 3 \\
\hline 1 & 2 & 6 & 0 & 3 & 4 & 5 \\
\hline 5 & 4 & 3 & 6 & 2 & 1 & 0 \\
\hline
\end{tabular}

\begin{tabular}{|l|l|l|l|l|l|l|}
\hline 0 & 1 & 2 & 3 & 4 & 5 & 6 \\
\hline 1 & 2 & 6 & 0 & 3 & 4 & 5 \\
\hline 3 & 0 & 1 & 4 & 5 & 6 & 2 \\
\hline 5 & 4 & 3 & 6 & 2 & 1 & 0 \\
\hline 2 & 6 & 5 & 1 & 0 & 3 & 4 \\
\hline 6 & 5 & 4 & 2 & 1 & 0 & 3 \\
\hline 4 & 3 & 0 & 5 & 6 & 2 & 1 \\
\hline
\end{tabular}




\section{E Maximum sets of pairwise orthogonal Youden rectangles}

E.1 An example of a 4-tuple of mutually orthogonal $5 \times 6$ Youden rectangles

\begin{tabular}{|l|l|l|l|l|l|}
\hline 0 & 1 & 2 & 3 & 4 & 5 \\
\hline 5 & 4 & 3 & 2 & 1 & 0 \\
\hline 4 & 5 & 1 & 0 & 3 & 2 \\
\hline 3 & 2 & 5 & 4 & 0 & 1 \\
\hline 2 & 0 & 4 & 1 & 5 & 3 \\
\hline
\end{tabular}

\begin{tabular}{|l|l|l|l|l|l|}
\hline 0 & 1 & 2 & 3 & 4 & 5 \\
\hline 4 & 5 & 1 & 0 & 3 & 2 \\
\hline 3 & 2 & 5 & 4 & 0 & 1 \\
\hline 2 & 4 & 3 & 1 & 5 & 0 \\
\hline 5 & 3 & 0 & 2 & 1 & 4 \\
\hline
\end{tabular}

\begin{tabular}{|l|l|l|l|l|l|}
\hline 0 & 1 & 2 & 3 & 4 & 5 \\
\hline 3 & 2 & 5 & 4 & 0 & 1 \\
\hline 5 & 0 & 4 & 2 & 1 & 3 \\
\hline 4 & 5 & 1 & 0 & 3 & 2 \\
\hline 1 & 4 & 3 & 5 & 2 & 0 \\
\hline
\end{tabular}

\begin{tabular}{|l|l|l|l|l|l|}
\hline 0 & 1 & 2 & 3 & 4 & 5 \\
\hline 2 & 0 & 4 & 1 & 5 & 3 \\
\hline 1 & 4 & 3 & 5 & 2 & 0 \\
\hline 5 & 3 & 0 & 2 & 1 & 4 \\
\hline 4 & 2 & 5 & 0 & 3 & 1 \\
\hline
\end{tabular}

E.2 An example of a 6-tuple of mutually orthogonal $3 \times 7$ Youden rectangles

\begin{tabular}{l}
\begin{tabular}{|l|l|l|l|l|l|l|}
\hline 0 & 1 & 2 & 3 & 4 & 5 & 6 \\
\hline 6 & 5 & 4 & 2 & 1 & 0 & 3 \\
\hline 2 & 6 & 5 & 1 & 0 & 3 & 4 \\
\hline
\end{tabular} \\
\begin{tabular}{|l|l|l|l|l|l|l|l|l|l|l|}
\hline 0 & 1 & 2 & 3 & 4 & 5 & 6 \\
\hline 5 & 0 & 1 & 4 & 5 & 6 & 2 \\
\hline 5 & 4 & 3 & 6 & 2 & 1 & 0 \\
\hline 5 & 4 & 3 & 6 & 2 & 1 & 0 \\
\hline
\end{tabular} \\
\hline
\end{tabular}

E.3 An example of a 6-tuple of mutually orthogonal $4 \times 7$ Youden rectangles

\begin{tabular}{|c|c|c|c|c|c|c|c|c|c|c|c|c|c|c|c|c|c|c|c|c|}
\hline 0 & 1 & \begin{tabular}{|l|}
2 \\
\end{tabular} & 3 & 4 & 5 & 6 & 0 & 1 & 2 & 3 & 4 & 5 & 6 & \begin{tabular}{|l|l}
0 \\
\end{tabular} & \begin{tabular}{|l|}
1 \\
\end{tabular} & 2 & 3 & 4 & 5 & \\
\hline 6 & 5 & 4 & 2 & 1 & 0 & 3 & 5 & 4 & 3 & 6 & 2 & 1 & 0 & 4 & 3 & 0 & 5 & 6 & 2 & \\
\hline 5 & 4 & 3 & 6 & 2 & 1 & 0 & 6 & 5 & 4 & 2 & $\begin{array}{ll} \\
\end{array}$ & 0 & 3 & $\overline{2}$ & 6 & 5 & 1 & 0 & 3 & 4 \\
\hline 4 & 3 & 0 & 5 & 6 & 2 & 1 & 2 & 6 & 5 & 1 & 0 & 3 & 4 & 3 & 0 & 1 & 4 & 5 & 6 & $\overline{2}$ \\
\hline & & & & & & & & & & & & & & & & & & & & \\
\hline 0 & 1 & 2 & 3 & 4 & 5 & 6 & 0 & 1 & 2 & 3 & 4 & 5 & 6 & \begin{tabular}{|l|l|}
0 \\
\end{tabular} & 1 & 2 & 3 & 4 & 5 & \\
\hline 3 & 0 & 1 & 4 & 5 & 6 & 2 & 2 & 6 & 5 & 1 & 0 & 3 & 4 & \begin{tabular}{|l|}
1 \\
\end{tabular} & 2 & 6 & 0 & 3 & 4 & 5 \\
\hline 1 & 2 & 6 & 0 & 3 & 4 & 5 & 4 & 3 & 0 & 5 & 6 & 2 & 1 & 3 & 0 & 1 & 4 & 5 & 6 & 2 \\
\hline 6 & 5 & 4 & 2 & 1 & 0 & 3 & 1 & 2 & 6 & 0 & 3 & 4 & 5 & 5 & 4 & 3 & 6 & 2 & 1 & 0 \\
\hline
\end{tabular}




\section{F Algorithms}

Here we give a more detailed description of the algorithms used.

\section{F.1 Extension Finding}

The algorithm to find all normalized non-isotopic triples of MOLR of size $k \times n$ is recursive and based on a breadth first search. It takes all normalized nonisotopic triples of MOLR of size $(k-1) \times n$ as input, finds an extension of size $k \times n$ and saves the result in a file. Note that nothing in the algorithm requires that the input rectangles are normalized, but in practice, we only used normalized rectangles as input.

It should also be noted that the algorithm will not necessarily produce normalized triples, and it may also produce many isotopic triples. The second part, described in the next subsection, picks out non-isotopic triples and outputs each such representative in normalized form.

The search when adding the $k$-th rows of the rectangles starts with the lexicographically largest permutation and goes to the smallest one, i.e., the search is done in reverse order.

Clearly, only a small fraction of all $n$ ! possible permutations can be used as the $k$-th row of the Latin rectangles. Thus, to speed up the algorithm in practice, for each triple of MOLR we generate three lists of permutations, $A_{k}$, $B_{k}, C_{k}$ that are possible as $k$-th rows of the corresponding Latin rectangles and use only those. For the purposes of keeping the pseudocode simple, this restriction is not reflected, and all permutations are taken from $S_{n}$, the full set of permutations on $n$ elements.

Pseudocode for the process of finding an extension of a triple of MOLR is given in Algorithm 1, 


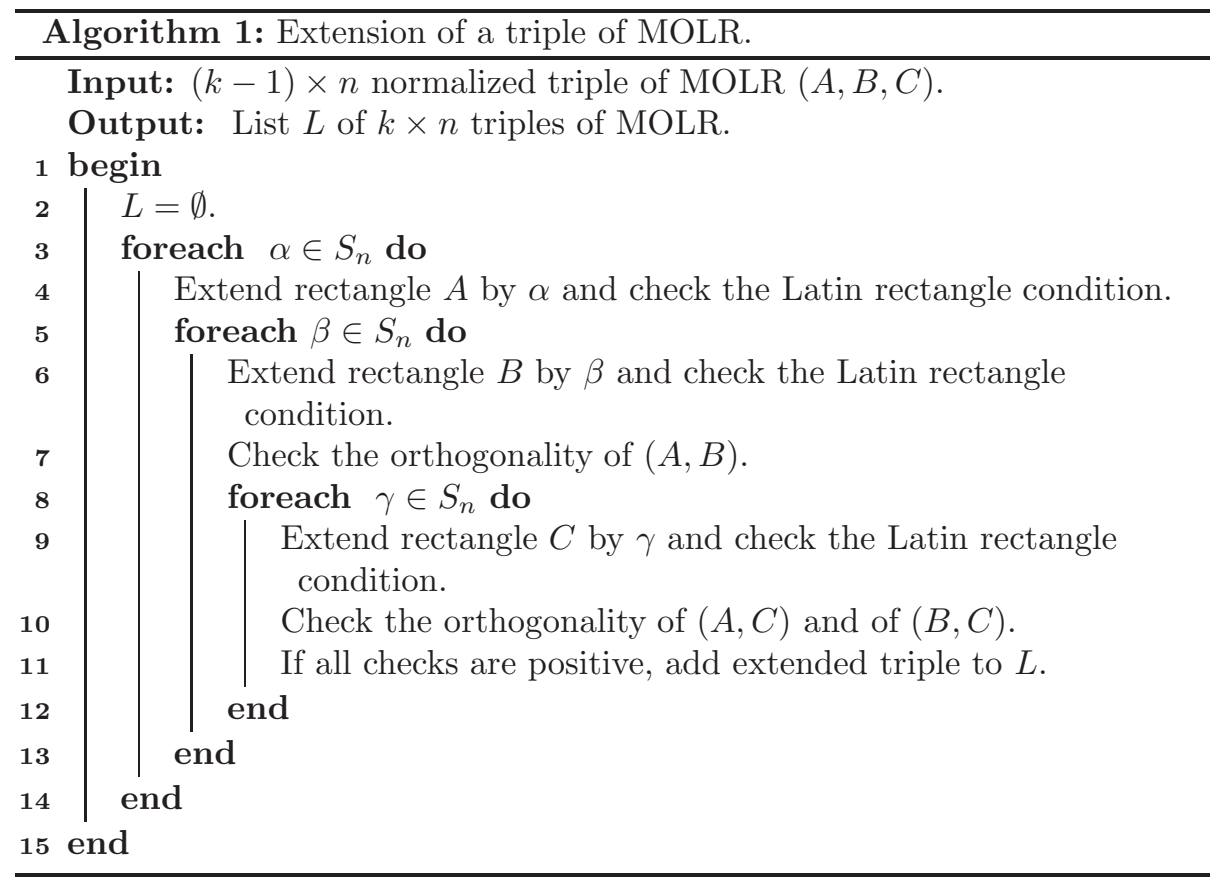

\section{F.2 Isotopism Rejection}

Algorithm 1 may create many isotopic triples of MOLR and the aim of our second step is to keep exactly one representative for each class of isotopic triples of MOLR, and to output that representative in normalized form. As the representative of an isotopism class we will choose the triple which is highest in the lexicographic order. The main idea for the isotopism rejection step is thus to check whether it is possible to transform a triple $(A, B, C)$ of MOLR to an isotopic triple with higher lexicographic order, and if that is possible, the triple $(A, B, C)$ is discarded. This test is applied to each generated triple in order to determine whether it should be output or not.

The isotopism rejection is based on all six types of permutations of a triple of MOLR of size $k \times n$, namely permuting rectangles, permuting rows, permuting columns, and permuting symbols separately in each rectangle. Therefore, in general, it requires six nested loops and $3 ! \times k ! \times n ! \times n !^{3}$ steps.

We use the concept of normalized triples of MOLR to reduce the complexity of this algorithm. First, the first rows are transformed to identity permutations by permuting symbols. As this transformation is unique once the column permutation $\sigma$ is chosen, we skip the loops with symbol permutations.

Second, since the Latin rectangles in a normalized triple of MOLR are ordered (condition (S2)), it is sufficient to choose which of the rectangles is the first one (three options) and reorder the last $k-1$ rows to satisfy condition (S3). 
The other two rectangles will be ordered by the second row.

Finally, rows are ordered by the first rectangle, therefore, we just need to choose which of the rows is the first one ( $k$ options). There is no restriction for permuting columns, so we keep this loop as it is. In summary, by using normalization, we have reduced the number of steps in the algorithm to $3 \times k \times n$ !.

Pseudocode for this step is given in Algorithm 2. In the pseudocode, $M_{2}$ denotes the second row of a matrix $M$, and the lexicographically largest permutation in a set $\alpha$ of permutations is denoted by $\max \{\alpha\}$.

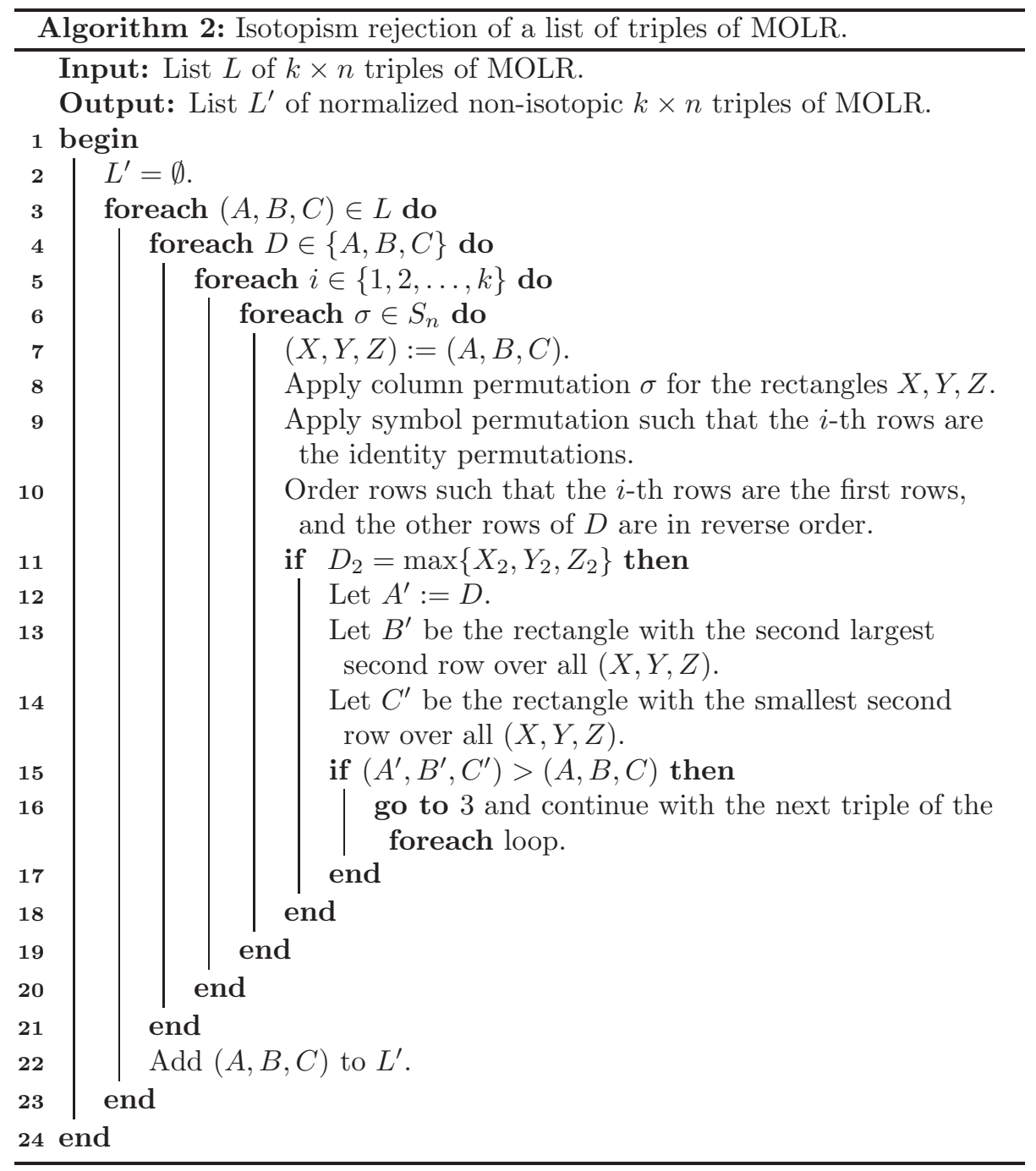

